\title{
Compétitivité et réseau public d'offre de soins : des Communautés Hospitalières de Territoire aux Groupements Hospitaliers de Territoire
} François FULCONIS ${ }^{1}$ Jérôme JOUBERT ${ }^{2}$

\section{Résumé}

La loi "Hôpital, patients, santé, territoires» (Hрsт) du 21 juillet 2009 a placé les démarches coopératives interorganisationnelles au cœur de son dispositif en créant la Communauté Hospitalière de Territoire $\left(\mathrm{C}_{\mathrm{H}}\right)$. Dans ce contexte, cet article présente les résultats d'une étude menée sur les caractéristiques organisationnelles d'une $\mathrm{C}_{\text {Hт. }}$ Il est mis en évidence comment la $\mathrm{C}_{\mathrm{HT}}$ est susceptible d'améliorer l'efficience et l'efficacité des acteurs publics sur un même territoire. Il est aussi souligné comment les insuffisances constatées des $\mathrm{C}_{\mathrm{HT}}$ sont à l'origine de l'apparition récente des Groupements Hospitaliers de Territoire ( $\left.\mathrm{GH}_{\mathrm{H}}\right)$.

\begin{abstract}
The law "Hospital, patients, health, territories" (July 2009) has placed inter-organizational collaborative approaches at the heart of its device. In this context, this article presents
\end{abstract}

1 François FULCONIS : Maître de Conférences en Sciences de Gestion à l'Université d'Avignon \& des Pays de Vaucluse (UAPV), il est membre permanent du CRETLOG (Université d'Aix-Marseille) et membre associé au LBNC (Université d'Avignon). Ses recherches portent sur le management des coopérations et des structures en réseau interorganisationnelles, le supply chain management et l'industrie de la prestation de services logistiques - francois.fulconis@univ-avignon.fr

2 Jérôme JOUBERT: Maître de Conférences en Sciences Économiques et membre du laboratoire LBNC de l'Université d'Avignon \& des Pays de Vaucluse (UAPV), il est chargé de missions auprès de la Direction Générale du Centre Hospitalier Intercommunal Aix-Pertuis (Chiap). Ses recherches portent principalement sur l'intégration régionale, les délocalisations et la compétitivité des territoires et des pays - jerome.joubert@univ-avignon.fr 
the results of a study on the organizational characteristics of a "Hospital Community of Territory" (Нст). We show how the Нст, causing pooling and reorganization of the health care supply, is likely to improve the efficiency and effectiveness of public actors on a territory. We highlighted how shortcomings of Нст are causing the recent appearance of a new institution: the "Hospital Groupings of Territory" (Hgt).

\section{Introduction}

Si les dépenses de soins constituent en France un sujet de plus en plus préoccupant, la véritable question ne réside-t-elle pas dans la réforme de l'organisation du système de santé bien plus que dans son financement tel qu'il est actuellement? C'est dans cet esprit que la loi «Hôpital, patients, santé, territoires» (HPST) du 21 juillet 2009 " portant réforme de l'hôpital et relative aux patients, à la santé et aux territoires» fut pensée. Présentée comme un "projet d'organisation sanitaire» et non pas comme un "projet de financement», cette loi vise à mettre en place une offre de soins gradués de qualité, accessibles à tous, satisfaisant à l'ensemble des besoins de santé. Dans cette perspective, cette loi a placé les démarches coopératives inter-organisationnelles au cœur de son objectif. Elle a créé pour cela la Communauté Hospitalière de Territoire ( pour en faire un outil dédié au renforcement des coopérations public/public. La CHT repose sur une convention librement conclue par les établissements publics de santé (Clément, 2012). Ainsi, même si son objectif consiste à réunir plusieurs établissements publics de santé appartenant à un même territoire, elle ne fut pas dotée de la personnalité morale.

Par la mise en œuvre d'une stratégie médicale commune, par une mutualisation de certaines fonctions ou par le transfert de compétences, la $\mathrm{CH}_{\mathrm{T}}$ avait pour finalité la recherche de la meilleure utilisation des ressources à disposition des établissements publics de santé et la complémentarité entre les acteurs.

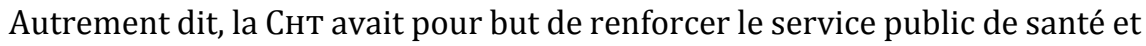
la qualité de prise en charge du patient en favorisant les coopérations entre établissements publics autonomes appartenant à une même zone géographique. Elle s'inscrivait en cela dans un long processus de territorialisation des politiques de santé publique, piloté et coordonné dorénavant par les Agences Régionales de Santé (ARs) en charge d'organiser, avec l'ensemble des acteurs concernés, l'offre régionale de soins. Elle s'appuyait également sur les enseignements tirés des limites des outils de coopération plus anciens reposant, par exemple, sur diverses conventions de partenariats, des Groupements d'Intérêt Public (GIP) ou des Groupements de Coopération Sanitaires (GCS).

Dans ce contexte, cet article présente les résultats d'une étude effectuée sur les caractéristiques organisationnelles de la Снт afin de mieux comprendre comment sa nature réticulaire est susceptible d'accroître la compétitivité de 
l'offre publique de soins en France en termes de parts de marché (préserver les actuelles ou en gagner de nouvelles). Autrement dit, cette compétitivité repose sur la capacité de la CHT à satisfaire à la fois des exigences d'efficience (rationalisation des moyens utilisés tout en améliorant la qualité et l'accessibilité des soins, augmentation de la rentabilité financière de ses membres sur le court terme, etc.) et d'efficacité (renforcer sa capacité à répondre aux besoins de santé sur le long terme). L'autre intérêt de cette étude est de montrer que les insuffisances et les dysfonctionnements relevés sur le cas analysé expliquent les raisons de la transformation actuelle des Снт en Groupements Hospitaliers de Territoire (GHT) selon l'Article 107 de la loi n 2016-41 du 20 janvier 2016 de «modernisation de notre système de santé». Dans une première partie, une réflexion sur l'origine et la place de la CHT en tant que nouvel acteur de la santé publique est proposée. À partir de la littérature existante sur la transformation actuelle du système hospitalier français et sur celle relative aux structures en réseau, quatre propositions théoriques sont formulées en vue de les confronter au terrain retenu. Dans une deuxième partie, les résultats les plus significatifs tirés de l'observation d'une CHT sont exposés. Ils confirment la nature réticulaire des CHT et posent la question de leur gouvernance. Dans une troisième partie, l'analyse de ces résultats donne à voir comment les insuffisances des CHT sont prises en compte dans la mise en place actuelle des GHT, et ce, tout en permettant une meilleure réponse aux besoins de santé.

\section{La CHT, une structure en réseau pour renforcer l'offre publique de soins}

Destinée à renforcer la compétitivité de l'offre publique de soins, la CHT est l'aboutissement d'une lente évolution caractérisée par l'intensification progressive des coopérations entre les établissements hospitaliers appartenant à un même territoire. La CHT est parallèlement une innovation institutionnelle destinée à accompagner la territorialisation des politiques de santé et à renforcer la compétitivité des acteurs publics (Fermon et Grandjean, 2015). En tant que regroupement d'acteurs, la littérature managériale sur les réseaux propose des cadres d'analyse pertinents pour décrire et mieux comprendre leur fonctionnement. Une grille d'observation est alors mobilisée pour analyser en profondeur une CHT.

\subsection{Du secteur au territoire: la $\mathrm{CH}_{\mathbf{H}}$, nouvel acteur de la santé publique}

L'émergence du territoire comme concept opérationnel de santé publique a été freinée par la centralité de l'hôpital public, généralement associé à une ville et baigné dans une tradition d'autarcie hospitalière peu propice aux coopérations et à l'émergence de stratégies territoriales (Amat-Roze, 2011). La territorialisation de la politique de santé publique démarre néanmoins en 1970 avec la planification sanitaire et l'identification de 256 secteurs. Fondée essentielle- 
ment sur une analyse quantitative des besoins et un principe d'égalité, cette «sectorialisation » vise un meilleur contrôle de l'offre de soins et du nombre de lits par la délivrance d'autorisations. Pilotée par le ministère de la Santé, cette approche nationale se régionalise progressivement. Comme le souligne Tabuteau (2010), la région devient le «cheval de Troie du pouvoir d'État» et se retrouve dans de nombreux acronymes utilisant la lettre «R » (SROS, ARH, URML, URCAM, CRCI, PrS, GRSP, etc.). Entre 1991 et 2011, trois générations de schémas régionaux d'organisation sanitaire (SROS) se succèdent. En 2003, la notion de territoire de santé remplace celle de secteur jusqu'alors privilégiée. Parallèlement, le diagnostic territorial, la concertation entre les acteurs et l'analyse des besoins deviennent la base de la planification sanitaire. La troisième génération de SRos est maintenant intégrée aux projets régionaux de santé (PRS), à côté d'un schéma de prévention et d'un schéma médico-social.

Des projets médicaux de territoire (РMT) sont élaborés par les ARs, pour organiser l'accessibilité, la graduation et la continuité des soins en tenant compte des bassins de vie et des habitudes des populations (Coldefy et Lucas-Gabrielli, 2010 et 2012). Le rôle de chaque établissement est défini dans les contrats pluriannuels d'objectifs et de moyens (СРОМ), signés entre l'ARs et les établissements, qui définissent des objectifs quantifiés en implantation d'équipement et en volume d'activité. À titre d'exemple, le Prs de la région Provence-Alpes-Côte d'Azur (PACA), distingue 133 espaces de proximité, 25 territoires de proximité et 9 de recours. La loi HPST de 2009 apparaît comme l'aboutissement de ce long processus. Elle accompagne la territorialisation des politiques de santé par une réforme de la gouvernance des hôpitaux et l'incitation à la coopération entre les acteurs par la création des CHт (Ruiller et Burellier, 2016). Ces dernières constituent une innovation institutionnelle qui a répondu aux quatre objectifs principaux suivants :

- donner naissance à un acteur territorial qui puisse décliner des politiques territoriales, l'idéal-type étant un territoire de santé suffisamment large pour regrouper différents niveaux de prise en charge réalisés de manière coordonnée par des offreurs regroupés en CHT (Baly et al., 2016). La CHT doit réunir des établissements publics d'un même territoire de santé capable de fournir les niveaux de soins de proximité et de recours infrarégional. L'objectif, partiellement atteint, étant de faire correspondre le découpage juridique en $\mathrm{CHT}$ avec le découpage territorial utilisé pour le pilotage administratif du PrS ;

- $\quad$ renforcer la compétitivité des établissements publics de santé en accroissant leur efficience et leur efficacité par une organisation juridique encourageant la mutualisation et la multiplication des partenariats, l'un des principaux objectifs étant de gagner des parts de marché sur leur territoire vis-à-vis du secteur privé (Grenier et Guitton-Philippe, 2011; Cazin, 2016) ;

- fournir une solution à des injonctions contradictoires de la politique de santé publique. Comme le souligne Grenier (2014), les acteurs publics 
sont soumis à des exigences contradictoires. Il leur est demandé des prises en charge individualisées et de proximité et, en même temps, des prises en charges performantes et efficientes qui reproduisent les meilleures pratiques. De même, d'un côté sont valorisées l'autonomie et la responsabilité des établissements, dotés seuls de la personnalité morale et recevant directement les financements, ce qui les pousse à la concurrence pour le partage d'une ressource commune (ONDAM). D'un autre côté, toutes les formes de coopération, de partage et de mutualisation sont encouragées. Le terme de « coopétition » trouve ici un riche terrain d'observation et de validation, la CHT étant alors le compromis organisationnel permettant sa mise en œuvre (Louazel et Keller, 2016) ;

- $\quad$ trouver le moyen de conserver et de soutenir de nombreux centres hospitaliers de taille modeste fragilisés par la T2A qui ne peuvent plus assumer tout seuls les multiples contraintes auxquelles ils doivent faire face (Le Menn et Milon, 2012). Citons pour mémoire les règlementations sur les seuils d'activité, les coûts croissants de certaines technologies, les contraintes liées à la démographie médicale et les évolutions de population défavorables au développement de l'activité. La contradiction entre la non viabilité, en l'état des règles financières et administratives actuelles, de nombreuses petites structures et le souhait de ne pas les faire disparaître comme acteurs autonomes fut aussi à l'origine de l'innovation juridique créant les CHT.

D’une philosophie peu intégrative et dotée d'une gouvernance fondée sur le consensus et l'égalité formelle entre les membres, les CHT vont rapidement montrer leurs limites, comme nous allons le voir par la suite, et être remplacées en 2016 par les GHT.

\subsection{Approche réticulaire des $\mathrm{CHT}_{\mathrm{H}}$}

À l'instar des structures en réseau très étudiées depuis les années 1980, l'analyse des CHT suppose de faire appel à des approches en termes de gestion de projet (projet médical commun inter-établissements), de mutualisation et de prise en charge partagée d'activités et de ressources, de pilotage transversal de fonctions (achats, systèmes d'information, ressources humaines, formations, gestion des flux de patients, logistique, etc.) et, plus largement, de management des (et par les) processus intra et inter-organisationnels (Sampieri-Teissier et Rollin, 2015). Plusieurs établissements sont en effet, sur un même territoire, mobilisés dans le but d'organiser et de coordonner leurs actifs, leurs ressources et leurs compétences nécessaires à la réalisation de leur projet médical commun. Dès lors, comment appréhender, à l'aide de la littérature, la mise en place de ce vaste projet? D'un point de vue théorique, l'approche que nous avons privilégiée se place résolument dans le domaine du management des relations inter-organisationnelles, plus spécialement dans les champs de l'économie industrielle et du management stratégique dans lesquels les structures en réseau y sont communément admises comme vecteur de compétitivité, c'est-à-dire 
comme une réponse organisationnelle à la recherche de facteurs d'efficience et d'efficacité (Snow et al., 1992 ; Voisin et al., 2004 ; Paché et Paraponaris, 2006 ; Suire et Vicente, 2008 ; Assens, 2013 ; Bironneau et Viviani, 2016).

Depuis les travaux initiés dans les années 1980 par des auteurs tels que Piore et Sabel (1984), Miles et Snow (1986) ou Thorelli (1986), la littérature sur les structures en réseau est abondante, allant d'organisations assez stables dans le temps (Fréry, 1998) à des organisations «virtuelles » (Aubert et al., 1999), ou encore « temporaires » avec une durée de vie très courte (Turner et Müller, 2003), voire «éphémères » (Salaun, 2014). Des tentatives d'élargissement du concept de «réseau clignotant» ont parallèlement été menées, notamment dans le cadre des pôles de compétitivité et des réseaux de santé (Bruyère et Verlaque, 2009). Dans le cas qui nous intéresse, celui des CHT, nous avons retenu une acception large des structures en réseau proche de celle de la notion de « cluster » au sens de Porter (2000), bien que l'innovation ne soit pas ici le but central de la $\mathrm{CHT}_{\mathrm{T}}$ qui relève d'ailleurs plutôt du principe de proximité que de celui de concentration. Nous nous inscrivons dans la même posture que Dussuc et Geindre (2015) qui, en reprenant la notion de «cluster », insistent sur la présence d'une pluralité d'entreprises ou d'acteurs associés dans un champ particulier, géographiquement proches, et qui tirent avantage de la présence les uns des autres.

Dès lors, nous retiendrons que les structures en réseau correspondent à la mise en œuvre de stratégies de coopération entre acteurs (entreprises, établissements, etc.) sur une même chaîne de valeur ajoutée. Qualifiés de partenaires, ces acteurs sont juridiquement et financièrement indépendants les uns des autres. Les multiples relations qu'ils entretiennent s'appuient sur une forte réciprocité d'intérêt et nécessitent un effort permanent de coordination pour éviter leur désagrégation. Suivant les principes d'élaboration des modèles d'analyse en sciences sociales proposés par Van Campenhoudt et Quivy (2011), on retrouve dans la littérature que les concepts de structure en réseau et de compétitivité ont pu être mis en relation pour procéder à l'élaboration du concept de «structures en réseau vecteur de compétitivité» (Fulconis, 2004). Dans ce dernier cas, cette élaboration repose sur l'identification de quatre composantes fondamentales «Hétérogénéité - Partenariat - Autonomie - Cohésion ». À partir de l'état de l'art sur l'approche réticulaire des organisations, en particulier des quatre composantes mentionnées précédemment, et de l'état de l'art sur le management hospitalier, nous avons élaboré la grille d'observation des CHT suivante (cf. Schéma 1). Les quatre composantes y sont traduites au moyen d'indicateurs (ou de caractéristiques) issus de la littérature. Ils nous permettent, d'une part, d'exprimer en termes directement observables le concept de «structures en réseau vecteur de compétitivité » et, d'autre part, d'observer empiriquement le fonctionnement d'un CHT. 


\section{Schéma 1 - Grille d'observation des Снt dans une perspective réticulaire}

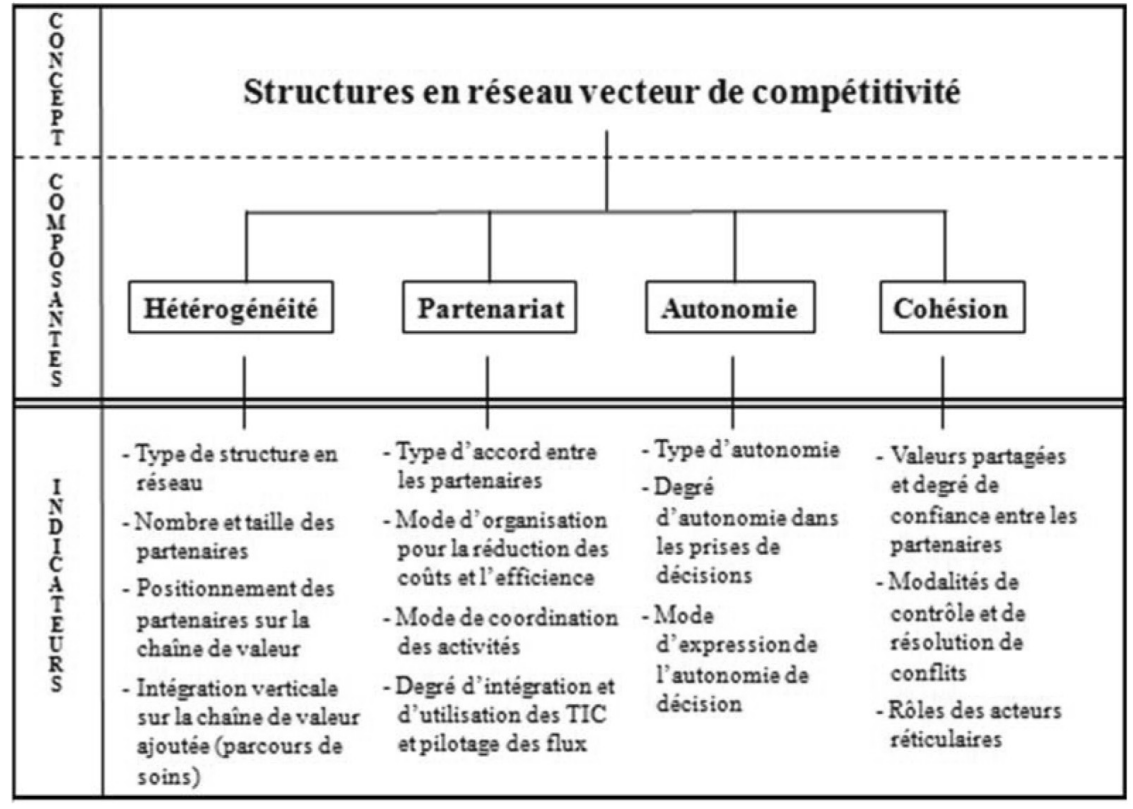

Source: Adapté de Fulconis (2004).

Cette grille d'observation constitue la trame du guide d'entretien utilisé lors de l'étude empirique. Ce guide a en effet servi d'outil pour recueillir et produire des données d'observation par la confrontation au terrain de quatre propositions théoriques ${ }^{3}$ générées à partir de la littérature et correspondant chacune à l'une des quatre composantes :

- $\quad$ PT 1 : par son hétérogénéité, la CHT est susceptible de contribuer à une meilleure maîtrise de la chaîne de valeur ajoutée associée à un parcours complet de soins ;

- $\quad$ Pт 2 : par le partenariat, la CHт est susceptible de faciliter, sur un même parcours de soins, la coordination des activités entre ses membres;

- $\quad$ Pт 3 : par l'autonomie, la CHT est susceptible de conjuguer indépendance et interdépendance de chacun de ses membres ;

- $\quad$ PT 4 : par la cohésion, la CHT est susceptible de maintenir uni, tout au long d'un processus de production de soins, chacun de ses membres.

$3 \quad$ Van Campenhoudt et Quivy (2011) rappellent que ces propositions «constituent en quelques sortes des réponses provisoires et relativement sommaires qui guideront le travail de recueil et d'analyse des données». 
Selon une démarche déductive, cette étape de conceptualisation et de formulation de propositions théoriques, indiquant les facteurs de compétitivité intrinsèques à la structure réticulaire, a permis de définir quels éléments prendre en considération pour étudier le fonctionnement d'une Снт particulière, la Снт-PP.

\subsection{Le cas de la Снt du Pays Provençal (Снt-Pि)}

Compte tenu de l'objet de notre investigation et partant d'un construit et de propositions théoriques à confronter au terrain, principalement par des entretiens, une recherche de type "qualitatif déductif» a été privilégiée (Hlady-Rispal, 2015). L'étude de cas a été choisie en tant que dispositif type de recherche (Wacheux, 1996 ; Giordano, 2003; Yin, 2009). Elle apparaît comme la stratégie la plus appropriée pour mieux comprendre comment la CHT-Pि a fonctionné du mois de janvier 2012 au mois d'avril 2016. En reprenant les travaux de Royer et Zarlowski (2014) sur la taille d'un échantillon pour un traitement qualitatif, deux raisons justifient ici le choix de l'étude de cas unique : elle permet d'observer un cas typique de Снт (la Снт-Pр était la première СHT, en taille, de la région PACA) et «d'identifier les circonstances et conditions» de sa mise en œuvre; elle permet aussi «de révéler un phénomène qui n'est pas rare» mais qui, par sa nouveauté, était jusqu'alors peu étudié par la communauté scientifique. De même, selon Gombault (2005), l'étude de cas unique se justifie ici par le fait qu'elle permet une investigation en profondeur et la compréhension de la spécificité du contexte organisationnel pour saisir les logiques d'acteurs. Quant aux frontières du cas (Miles et Huberman, 2003), la Снт-Pp était facile à délimiter puisqu'elle regroupait quatre établissements (cf. Schéma 2) : le centre hospitalier de Digne-les-Bains et celui de Manosque (Alpes-de-Haute-Provence), le centre hospitalier intercommunal Aix-Pertuis (Bouches-du-Rhône et Vaucluse) et le centre hospitalier de Salon-de-Provence (Bouches-du-Rhône). L'étude de leur comportement et de leurs interactions constituent le niveau d'analyse de la recherche. 


\section{Schéma 2 - Représentation de la Chт-Pр}

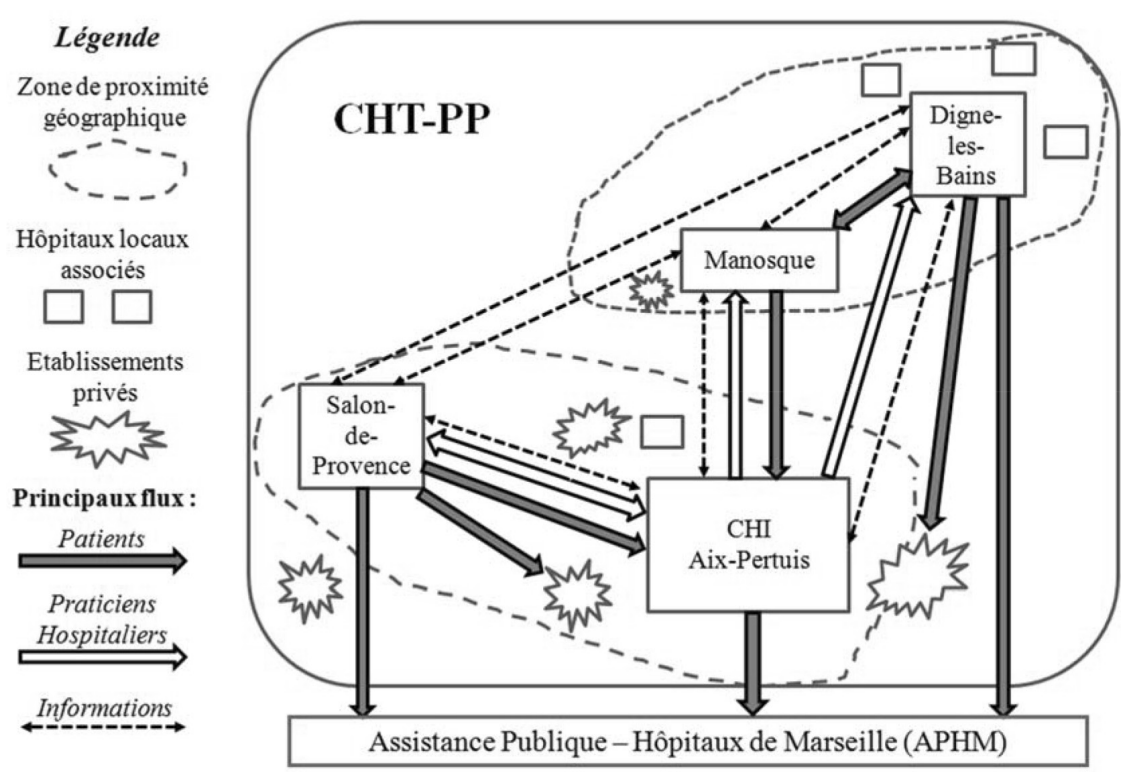

Conformément à la caractérisation de la démarche contextualiste (Warnotte et Rousseau, 1993 ; Husser, 2005), l'étude du cas de la CHт-Pp nécessite d'analyser «la dynamique organisationnelle dans le contexte qui l'entoure» (Husser, 2005), tant au niveau vertical par l'étude de facteurs internes et externes à un établissement hospitalier public (statut des personnels et diversité des mondes sociaux, règles d'organisation interne et de gouvernance), qu'au niveau horizontal par l'analyse des séquences interconnectées des règles de la coopération inter-établissements hospitaliers (GIP et Gcs, puis ChT suivies des GHT). Ce dernier point renvoie au second principe de la démarche contextualiste qui a pour vocation d'étudier un processus organisationnel dans son rapport au passé, au présent et au futur (Husser, 2005).

La démarche retenue pour recueillir les données s'appuie principalement sur l'observation de cette $\mathrm{CHT}_{\text {T }}$ depuis sa création. Cette phase d'observation repose sur l'exploitation de sources primaires et secondaires. Les sources primaires ont été recueillies sur une période de quatre ans (2012 à 2015), dans le cadre d'une Convention de recherche liant le Centre Hospitalier Intercommunal AixPertuis (CHIAP) et le laboratoire de recherche en Droit, Économie et Gestion de l'université d'appartenance des auteurs. Au total, plus d'une cinquantaine d'entretiens en face-à-face ont été menés avec des responsables et des acteurs concernant la CHT-PP (cf. Tableau 1). Parallèlement, en 2014 et 2015, un suivi d'équipes de direction nous a offert la possibilité de participer aux réunions des différentes instances du CHIAP : directoire, conseil de surveillance, Commissions Médicales d'Établissements (СмE), Comités Techniques d'Établissements (СТE) et aux réunions de la CHт. Cette participation a permis un riche recueil de 
données empiriques grâce à l'observation des acteurs (observation non participante et échanges informels avec des responsables).

\section{Tableau 1 - Caractéristiques des répondants concernant la Снt-Pि}

\begin{tabular}{|l|l|c|}
\hline \multicolumn{1}{|c|}{ Fonctions } & \multicolumn{1}{c|}{ Appartenances } & Nombres \\
\hline $\begin{array}{l}\text { Les Directeurs généraux et les pré- } \\
\text { sidents de CME }\end{array}$ & $\begin{array}{l}\text { CHIAP, Salon-de-Provence, Manosque } \\
\text { et Digne-les-Bains }\end{array}$ & 8 \\
\hline La totalité des membres du directoire & CHIAP & 10 \\
\hline La totalité des chefs de pôles & CHIAP & 11 \\
\hline Les directeurs adjoints & CHIAP et Digne-les-Bains & 14 \\
\hline $\begin{array}{l}\text { Des cadres supérieurs de santé et des } \\
\text { praticiens hospitaliers }\end{array}$ & $\begin{array}{l}\text { CHIAP, Salon-de-Provence, Manosque } \\
\text { et Digne-les-Bains }\end{array}$ & 10 \\
\hline La présidente & CSIRMT du CHIAP & 1 \\
\hline Un directeur territorial & ARS PACA & 1 \\
\hline
\end{tabular}

Les sources secondaires ont été utilisées comme des données confirmatoires ou infirmatives. Elles sont aussi venues compléter des aspects souvent trop récents pour être approfondis dans les entretiens. Ces sources sont principalement constituées par la législation et les décrets d'application, les rapports de l'Inspection Générale des Affaires Sociales (IGAS) et les programmes cadres ministériels (programmes nationaux et stratégie nationale de santé), les SRos, les Contrats Pluriannuels d'Objectifs et de Moyens (СРОМ), les projets d'établissement, les relevés de décisions des instances hospitalières. Dans un souci de triangulation des données, en plus de ces documents, des informations disponibles dans la presse économique et dans la littérature ont également été étudiées.

L'analyse qualitative des données empiriques a été réalisée à partir d'analyses de contenu des entretiens retranscrits et des documents évoqués selon les méthodes en vigueur (Gavard-Perret et al., 2012 ; Dumez, 2013; Thiétart et al., 2014). Ces analyses ont eu lieu pendant (fiches de synthèse d'entretiens et de documents) et après la période de recueil des données. Dans le cadre d'une étape de pré-analyse, une lecture flottante portant sur l'ensemble des données recueillies a permis d'opérationnaliser plus avant les quatre propositions théoriques. Sur la base de cette pré-analyse, le codage a fait émerger des sous-thèmes permettant d'explorer ces propositions. Leur synthèse a permis d'aboutir aux résultats de la recherche. Notons qu'en plus de la triangulation et de la contextualisation des données (Yin, 2009), mais aussi de la saturation empirique du terrain (collecte des données jusqu'en 2016 n'apportant in fine que des informations marginales ne remettant pas en question les cadres construits) (Grawitz, 2000 ; Thiétart et al., 2014), la validité interne de cette recherche fut en outre confortée par la soumission des résultats, pour validation lors de séances de restitution, à des acteurs clés (présidents de CME et chefs de pôles) de la Снт-PP. Enfin, la validité de notre recherche (interne et externe) 
est renforcée par l'adéquation entre les dispositions réglementaires de la loi du 26 janvier 2016 de «modernisation de notre système de santé» créant les GHT et les insuffisances constatées d'une Снт particulière au travers de notre grille d'observation.

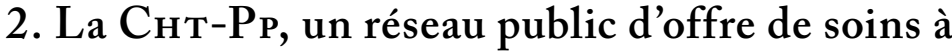 gouverner}

Les résultats les plus significatifs issus de l'observation de la CHт-Pp sont ici présentés. Ils reposent sur la confrontation au terrain des quatre propositions théoriques énoncées précédemment. À l'aide des composantes Hétérogénéité, Partenariat, Autonomie et Cohésion, ces résultats confirment que les CHT sont des structures en réseau spécifiques, décrivent leur fonctionnement et posent la question de leur gouvernance.

\subsection{L'Hétérogénéité de la $\mathrm{CH}_{\mathrm{H}}-\mathrm{Pp}$}

Comme il l'est souvent souligné dans la littérature (Ehlinger et al., 2007), la présence d'une firme dominante est plutôt un atout. Cette « hub firm », «firme pivot» ou encore «broker», peut jouer le rôle de centre stratégique. Siège de la $\mathrm{CHT}_{\mathrm{T}}$ et hôpital de référence, le CHIAP apparaît comme la "hub firm» avec une responsabilité primordiale en termes de stratégie et de coordination du réseau. Il doit le faire en exerçant une forme de «soft power » (North, 1990) fondé sur des compétences professionnelles et non hiérarchiques. Il est notamment attendu de la firme pivot un rôle moteur dans la définition des termes de la «convention de gouvernance», dans ces dimensions de «sens», de «partage» et de «transparence» (Carles, 2006). L'autre atout d'un groupe asymétrique réside dans la possibilité, en faisant appel à la théorie des biens collectifs (Kindleberger, 1986), que l'« hégémon » (ici la « hub firm») puisse prendre à sa charge le coût de production d'un ensemble de biens collectifs. La CHT-Pp constitue donc un réseau asymétrique centré autour d'un hôpital de référence (le CHIAP) et de trois hôpitaux de plus petite taille. Cette notion d'hôpital de référence, dont le contenu et la légitimité ne sont pas reconnus par le législateur dans le cadre de Снт (mais le deviennent dans le projet de GHT, Loi 2015, par la notion «d'établissement support»), signifie seulement qu'un des établissements est choisi dans la convention constitutive pour être le siège des organes politiques de la CHT. Cela ne lui accorde explicitement aucun pouvoir particulier. Il en reste néanmoins vrai que l'hôpital d'Aix-Pertuis est la structure dominante de la CHT, l'hôpital de référence de fait.

L'hétérogénéité au sein d'une CHT se mesure par la taille, l'activité globale, l'ampleur et la diversité de l'offre de soins, la situation financière, l'environnement économique et le bassin de population. Le Tableau 2 précise l'hétérogénéité des quatre établissements composant la CHT-PP. 
Tableau 2 - La Cht-Pp : un ensemble d'établissements hétérogènes

\begin{tabular}{|c|c|c|c|c|}
\hline Indicateurs & Aix-Pertuis & $\begin{array}{l}\text { Salon-de- } \\
\text { Provence }\end{array}$ & Manosque & $\begin{array}{l}\text { Digne-les- } \\
\text { Bains }\end{array}$ \\
\hline Nombre RSA MCO & 29762 & 14384 & 10592 & 7105 \\
\hline Médecine & 19615 & 9923 & 7254 & 4631 \\
\hline Chirurgie & 6899 & 2674 & 2247 & 1753 \\
\hline Obstétrique & 3248 & 1787 & 1091 & 721 \\
\hline Recettes totales en K€ & 191165 & 76173 & 56829 & 73963 \\
\hline Nombre GHS recours & $3,4 \%$ & $0,7 \%$ & $0,7 \%$ & $1,3 \%$ \\
\hline Bassin en habitants & 551133 & 154457 & 104597 & 61120 \\
\hline Dette/Recettes (millions €) & $63 / 196$ & $21 / 76$ & $73 / 56$ & $44 / 73$ \\
\hline Taux de CAF (Brut/Net) & $5,1 / 40$ & $4,9 / 59$ & $7 / 25,2$ & $5,8 /-108$ \\
\hline
\end{tabular}

Source: Données HospiDiag 2013 (Url: http://hospidiag.atih.sante.fr consultée le 16/02/2016).

L'hôpital d'Aix-Pertuis représente 48 \% de l'activité mesurée en nombre de Résumés de Sortie Anonymes (RSA) et emploie plus de 50 \% des anesthésistes. Certains établissements n'assument que des activités de Médecine, Chirurgie, Obstétrique (Mco) alors que d'autres, comme Digne-les-Bains, intègrent un secteur psychiatrique important et plusieurs hôpitaux locaux. Les hôpitaux ont une structure d'offre que l'on observe au travers de leur « casemix ». Elle fait apparaître de nombreuses activités similaires même si l'hôpital d'Aix-Pertuis est le seul à présenter un nombre significatif de groupes homogènes de malades (GHM) recours. Plutôt concurrents, on n'observe pas vraiment une spécialisation des activités de soins leur attribuant un positionnement bien différent dans la chaîne de valeur du parcours de soins et dans la graduation des soins. De plus, lors d'un épisode de soins, les mouvements des patients intra-CHT sont assez faibles.

L'hétérogénéité des membres semble aussi limitée quant à leur fonctionnement. Modes de financement et instances de direction (directoire, conseil de surveillance, CME, CTE, etc.) sont identiques et fortement encadrés par la loi. Les personnels sont issus des mêmes corporations professionnelles (soignants, médecins, corps de direction) dont les carrières et les statuts sont gérés nationalement.

Cette hétérogénéité est également limitée dans son ampleur pour deux raisons :

- $\quad$ elle n'intègre pas des offreurs publics comme le CHU ou des établissements spécialisés (PSY et SSR). La CHT-PP ne peut pas offrir certains soins de recours régionaux comme les transplantations et la neurochirurgie, ni organiser en son sein des prises en charge de longue durée ou spécialisées ;

- $\quad$ elle ne contient pas les établissements privés ni la médecine de ville qui constituent pourtant des acteurs importants dans les soins de premiers recours et dans la chirurgie ambulatoire notamment. 
En synthèse, par son hétérogénéité limitée, la СнT-Pp ne peut proposer qu'une prise en charge incomplète sur son territoire des patients et de leurs pathologies.

\subsection{Le partenariat au sein de la $\mathrm{CHT}_{\mathrm{H}}-\mathrm{PP}$}

La Chт-Pp résulte d'un accord volontaire, la Convention constitutive, qui organise la gouvernance du regroupement et facilite la multiplication des partenariats. Au sein de la CHT-Pr, les établissements membres peuvent utiliser les 17 instruments juridiques de coopération à disposition des $\mathrm{CHT}_{\text {Tépertoriés par }}$ l'ANAP (2011). La ChT-Pp utilise des modes organiques comme les Gcs, ou le mode fonctionnel par l'intermédiaire de conventions portant sur l'utilisation de ressources communes en équipement et en personnels. Les objectifs de ces partenariats peuvent être regroupés en trois domaines : recherche d'efficience et de compétitivité des membres par la mutualisation et le partage des coûts; réorganisation des filières de soins pour une meilleure coordination au service de la prise en charge des malades ; et convergence des systèmes d'information pour un usage renforcé des outils numériques et un pilotage commun des flux de patients.

La multiplication des coopérations visant l'efficience sont une des conséquences d'un système de financement fondé sur la comparaison des coûts de prise en charge des GHм. La Cнт-PP a mis en œuvre un ensemble de conventions visant à gérer en commun ces activités et les ressources rares qu'elles requièrent. Un certain nombre de recrutements partagés ont été effectués (ingénieur biomédical, ingénieur phare pour les achats, radio-physicien sur les quatre sites, plateau juridique commun) et l'hôtellerie et la formation sont gérées par un GCS auquel participe des membres extérieurs à la CHT-PP. La mise en place d'une garde de territoire en imagerie est en cours et les audits en biologie et en finance, préalable à la certification, sont conduits en commun pour diminuer les coûts et favoriser les rapprochements. Compte tenu des distances entre les membres, l'utilisation commune de gros équipements est rendu difficile, alors que les partenariats avec le secteur privé sur des logiques de sites sont plus fréquents.

Les partenariats portant sur les filières de soins cliniques sont plus récents et concernent directement le cœur du métier. Au niveau de la CHT-PP, des ressources médicales sont mises en commun pour le complexe anesthésie réanimation et des consultations avancées sont réalisées en pédiatrie et neurologie ainsi qu'en chirurgie ORL et urologique (Cazin, 2016). Des spécialisations croisées d'activité se mettent également en place progressivement entre les établissements du réseau. Sur une activité de gastro-entérologie, une compétence d'un des membres est consolidée par la décision d'un autre de ne pas proposer une offre concurrente. Sur un même parcours de patients en cancérologie, deux membres géographiquement proches se spécialisent, l'un sur la chimiothérapie, l'autre sur la chirurgie. Ces initiatives restent ponctuelles et délicates à mettre en œuvre car elles supposent un abandon réciproque d'activité qui peut venir 
à court terme heurter la logique financière. "Pourquoi renoncer à une activité rentable? ? est une remarque parfois entendue comme la peur de perdre des patients et des activités. Elles reposent également sur des efforts de mobilité et d'adaptation des praticiens hospitaliers et des personnels soignants.

Depuis 2011, l'hôpital numérique est un mot d'ordre ministériel et un vaste programme de renforcement de l'utilisation du numérique dans les établissements de santé. En la matière, les coopérations avancent lentement au sein de la Снт-Pp car les logiciels ne sont pas toujours compatibles, les départements d'information médicale relèvent de la direction de chaque établissement. Des progrès ont été obtenus grâce à la mise en place d'une direction commune entre deux établissements qui procède dorénavant à des arbitrages en matière d'achat informatique et favorise la collaboration des professionnels des DIM concernés. Mais l'identification unique des patients et le dossier médical partagé (DMP) restent à mettre en place. Les parcours multi-établissements dans le cadre d'une prise en charge commune et concertée par les établissements de la CHT-Pp restent rares. Comme le souligne un cadre de direction : "Il n'y a pas de véritable pilotage commun des flux mais une gestion laborieuse des interfaces ». Dans ce domaine aussi, le principe confédératif et l'absence de hiérarchie à la base de la création des CHT montrent leurs limites et ne provoquent pas des partenariats d'une ampleur suffisante.

En somme, les partenariats entre les membres du réseau sont multiples et avaient commencé bien avant la création de la CHT-PP. Celle-ci a légèrement accéléré le processus. Ils restent malgré tout désordonnés, contextualisés, partiels, réversibles et à géométrie variable.

\subsection{L'autonomie au sein de la $\mathrm{CH}_{\mathrm{t}}-\mathrm{Pp}$}

Un réseau d'acteurs indépendants, comme l'est une CHT, ne doit pas être confondu avec une entreprise multinationale fonctionnant en réseau d'établissements coordonnés et hiérarchisés. À la base du fonctionnement de la CHT-PP, comme pour toutes $\mathrm{CHT}$, il y a une égalité juridique des membres. L'article 2 de la Convention constitutive de la CHT-PP du 17 Janvier 2012 précise que la stratégie commune s'effectue «dans le respect de l'identité de chaque établissement».

Le degré d'autonomie y est élevé, tous les établissements sont des personnes morales, ils emploient directement leur personnel et possèdent les mêmes organes délibératifs et décisionnaires. Ils disposent d'un budget propre, d'une dette propre. Les recettes leurs sont attribuées pour faire face à l'ensemble de leurs dépenses. Les établissements gèrent le dialogue social, définissent leur organisation et le découpage en pôles d'activité, construisent leur projet médical, décident des recrutements comme de l'ouverture ou de la fermeture de nouvelles activités. En reprenant les concepts de Williamson (1981), ce n'est ni la hiérarchie, ni le marché qui est le principe moteur de la СНт mais l'influence, le partage, la réciprocité. 
La capacité à prendre des décisions importantes et à effectuer des choix stratégiques, sans passer par l'approbation des autres membres et des organes de la CHT-Pp, est un mode d'expression caractéristique de cette autonomie. À titre d'exemple, un membre de la СНт-Pि souhaite entretenir un partenariat privilégié avec le secteur privé présent dans sa ville, un autre souhaite maintenir des liens forts avec le Centre Hospitalier Universitaire ( $\mathrm{CHU}$ ) de proximité. Ces comportements stratégiques ne vont pas forcément dans le sens des intérêts commun de la Снт-Pि, mais ils attestent du fait qu'il s'agit d'un regroupement de membres autonomes défendant des intérêts propres.

Le pari de la CHT en général, c'est que l'autonomie sous influence peut constituer la meilleure solution, notamment par rapport aux alternatives que sont la fusion ou la mise sous tutelle. Un des moyens utilisés pour susciter cette influence est, dans le cas de la СНT-PP, la mise en place d'une direction commune entre Aix-Pertuis et Digne-les-Bains. Cette configuration institutionnelle semble la meilleure façon de gérer la dialectique dépendance-interdépendance. Il est souligné qu'elle ne doit pas être vécue comme une mise sous tutelle d'un petit établissement fragile par un plus grand centre hospitalier, mais comme un moyen de maintenir les atouts de l'autonomie sans en subir les inconvénients. Les deux hôpitaux ont chacun un directeur, même s'ils disposent de deux administrations distinctes. Selon les membres de la direction du Chiap rencontrés, ce dispositif se révèle être un gage de cohérence des choix et de bonne coordination, tout en permettant la prise de décisions qui doivent tenir compte des spécificités locales afin de faciliter leur mise en œuvre opérationnelle.

Il ressort également de l'étude de terrain, que l'autonomie des établissements est bien plus contrariée par des acteurs externes à la CHT-PP tels que l'ARs, l'HAS, la CNAM, la CRC et la DGOS, que par les autres membres de la CHT. Les autorisations d'activité, les mesures pour le retour à l'équilibre financier, les décisions majeures d'investissements, sont soumises à ces autorités externes et non pas négociées en interne.

\subsection{La cohésion de la Cht-PP}

Pendant la vie du réseau, depuis sa création jusqu'à son éventuelle disparition, le niveau de cohésion apparaît comme un facteur fondamental de réussite. Celui-ci dépend d'un certain nombre de facteurs tels que l'existence de valeurs partagées et le degré de confiance entre les membres, les modalités de contrôle et de résolution de conflits et le rôle des acteurs réticulaires.

La structure de gouvernance de la CHт-Pp est assez encadrée par la loi. La cohésion est normalement maintenue par le haut grâce aux trois assemblées : celles des directeurs, celles des présidents de CME et celles des présidents de conseil de surveillance qui sont les lieux de contrôle et de résolution des problèmes. La Convention constitutive impose un projet médical commun et demande une mise en cohérence de leurs documents stratégiques propres: «contrats pluriannuels d'objectifs et de moyens, projets d'établissement, plans 
globaux de financement pluriannuels et programmes d'investissement des établissements ». Le bon fonctionnement de ces assemblées, qui ont un faible pouvoir décisionnel, dépend de la qualité des relations personnelles et de la confiance entre les acteurs réticulaires constitués par les chefs de pôles, les présidents de CME, les directeurs et les grands élus locaux. Cette cohésion est également maintenue en externe par l'ARs PACA dont le DG est le supérieur hiérarchique des directeurs d'établissements et qui disposent de fonds d'intervention régionaux (FIR) dédiés à la coopération.

La réciprocité et la confiance entre ces différents acteurs de la CHT-PP ne sont ni évidentes, ni automatiques, même si la volonté de remplir au mieux les missions de service public crée une maïeutique indispensable au maintien de la cohésion du réseau par-delà les intérêts de court terme de chacun de ses membres. Pour la CHT-PP, la cohésion est également renforcée par la multiplication d'espaces de rencontres entre les professionnels qui permettent un partage d'expériences sur les pratiques. Les membres participent ensemble aux RMM, Clud, Clin, Clan, Csth, Covras et Comedims ${ }^{4}$, mais aussi à des Réunions de Concertation Pluridisciplinaires (RCP) communes et d'Évaluations des Pratiques Professionnelles (EPP). La cohésion autour des projets communs reste fragile car elle est très dépendante des relations interpersonnelles entre les praticiens hospitaliers, souvent issus des mêmes facultés et intervenant ensemble sur les mêmes parcours de soins. Comme le déclare un directeur-adjoint : «on monte de bons projets, mais le départ d'une personne remet tout en cause».

Tous les membres d'un réseau, dans la mesure où celui-ci est constitué sur la base du volontariat, doivent s'y retrouver. Dans ces conditions, la cohésion suppose qu'ils estiment tous profiter de leur appartenance au réseau. Le risque est que la CHT soit perçue, non pas comme mutuellement bénéfique, mais comme parasite, au mieux commensale, au pire pathogène. Dans le cas d'une CHT asymétrique, cela peut poser problème pour les membres les plus fragiles qui peuvent légitimement craindre que la CHT soit la modalité la plus efficace, après la disparition ou la fusion, pour supprimer des activités. C'est toute la difficulté de l'hôpital d'Aix-Pertuis qui peut légitimement tirer profit de la CHT mais sans que cela se fasse au détriment des autres. La spécialisation et l'abandon d'activités non rentables étant inévitables, il est important, comme le souligne le directeur du CHIAP : «d'insister sur les gains que les membres fragiles retirent de la CHT : réduction de leur vulnérabilité, mise à disposition d'outils, d'expertise et de professionnels en provenance des autres établissements ».

4 Rмm : revue de morbidité et de mortalité; CLuD : comité de lutte contre la douleur; CLIN : comité de lutte contre les infections nosocomiales; CLAN : comité de liaison en alimentation et nutrition; Csth : comité de la sécurité transfusionnelle et de l'hémovigilance ; Covras : comité des vigilances et des risques associés aux soins; Comedims : commission du médicament et des dispositifs médicaux stériles. 


\section{Discussion : les CHт, une étape intermédiaire mais nécessaire}

L'analyse des caractéristiques organisationnelles de la CHT-PP, grâce aux composantes «Hétérogénéité - Partenariat - Autonomie - Cohésion », a permis de préciser son fonctionnement en réseau. Nous avons également pu identifier un ensemble de difficultés et d'insuffisances au niveau de chacune des quatre composantes qui entravent sa mise en œuvre et limite la performance économique d'ensemble du réseau. Nous allons voir comment ces insuffisances sont prises en compte par la création récente des GHT et ce que l'on peut en attendre en termes de compétitivité du nouveau groupement ainsi constitué. Cette nouvelle forme institutionnelle va dans le sens d'une organisation plus intégrée: la reconnaissance officielle de l'hôpital référent et une dévolution obligatoire d'un ensemble de fonctions supports, la construction et la réalisation d'un projet médical partagé impératif, l'obligation de réaliser ensemble les activités médico-techniques et la couverture totale du territoire par l'adhésion obligatoire de tous les établissements à un seul GHT. Elle élargit également les regroupements aux CHU et aux établissements publics spécialisés du territoire. Aujourd'hui, les 135 GHT restent cependant des acteurs parmi d'autres du service public territorial auquel participent également les cliniques privées et la médecine de ville.

\subsection{Des Cht vers les Ght : un changement profond du cadre juridique}

Toutes les composantes sont affectées par les modifications introduites dans la loi du 26 janvier 2016 créant les GHT. Comme le montre le Tableau 3 ci-dessous, par rapport aux CHT, l'hétérogénéité est augmentée, les partenariats sont organisés et multipliés, le cadre de l'autonomie est modifié et la cohésion est stabilisée. 


\section{Tableau 3 - Une comparaison $\mathrm{CH}_{\mathrm{T}}$ vs $\mathrm{GHT}$}

\begin{tabular}{|c|c|c|}
\hline $\begin{array}{l}\text { COMPOSANTES ET } \\
\text { INDICATEURS }\end{array}$ & $\begin{array}{c}\text { Снт-PP } \\
\text { (Observation terrain) }\end{array}$ & $\begin{array}{c}\text { GHT } \\
\text { (Analyse de la loi santé 2016) }\end{array}$ \\
\hline HÉTÉROGÉNÉITÉ & Limitée & Augmentée \\
\hline $\begin{array}{l}\text { Type de structure en } \\
\text { réseau }\end{array}$ & $\begin{array}{l}\text { Réseau maillé avec égalité for- } \\
\text { melle des membres. }\end{array}$ & $\begin{array}{l}\text { Réseau en étoile de type centre } \\
\text { périphérie avec reconnaissance } \\
\text { de l'hôpital pivot. }\end{array}$ \\
\hline $\begin{array}{l}\text { Nombre et taille des } \\
\text { partenaires }\end{array}$ & $\begin{array}{l}\text { Nombre de membres réduit. } \\
\text { Adhésion en principe volontaire. }\end{array}$ & $\begin{array}{l}\text { Nombre de membres élevé. } \\
\text { Adhésion obligatoire et décou- } \\
\text { page imposé par l'ARs. }\end{array}$ \\
\hline $\begin{array}{l}\text { Positionnement des } \\
\text { partenaires }\end{array}$ & $\begin{array}{l}\text { Domaines Mco exclusivement } \\
\text { avec duplications nombreuses. } \\
\text { Absence aux deux extrémités de } \\
\text { la chaîne de valeur. Redondance } \\
\text { des activités. }\end{array}$ & $\begin{array}{l}\text { Les } 4 \text { (Mco, SsR, Psy et HAD) } \\
\text { champs sont concernés. Les ex- } \\
\text { trémités sont incluses (CHU et } \\
\text { médico-sociale). }\end{array}$ \\
\hline $\begin{array}{l}\text { Intégration verticale } \\
\text { sur la chaîne de } \\
\text { valeur }\end{array}$ & $\begin{array}{l}\text { Mouvement faible des patients } \\
\text { à l'intérieur du réseau (entre les } \\
\text { établissements lors d'un parcours } \\
\text { de soins). }\end{array}$ & $\begin{array}{l}\text { Prise en charge, au sein du GHT, } \\
\text { de l'intégralité d'un parcours de } \\
\text { soins favorisé par l'hétérogénéité } \\
\text { et la spécialisation des membres. }\end{array}$ \\
\hline PARTENARIAT & Désordonnés et facultatifs & Nombreux et cartographiés \\
\hline $\begin{array}{l}\text { Type d'accord entre } \\
\text { les partenaires }\end{array}$ & $\begin{array}{l}\text { Type confédératif. Coopération } \\
\text { possible et encouragée dans } \\
\text { plusieurs domaines et couverture } \\
\text { partielle du territoire national. }\end{array}$ & $\begin{array}{l}\text { Type fédératif. Coopération im- } \\
\text { posée dans un domaine précis. } \\
\text { Couverture totale du territoire } \\
\text { national. }\end{array}$ \\
\hline $\begin{array}{l}\text { Mode d'organisation } \\
\text { pour la réduction des } \\
\text { coûts et l'efficience }\end{array}$ & $\begin{array}{l}\text { Assemblage ex post de projets } \\
\text { d'établissements, coopération } \\
\text { empirique selon la bonne volonté. } \\
\text { Possibilités de fédération-médi- } \\
\text { cales inter-hospitalières faible- } \\
\text { ment utilisées. }\end{array}$ & $\begin{array}{l}\text { Impartition des activités basée } \\
\text { sur le projet médical partagé } \\
\text { (PMP). } \\
\text { Mutualisation obligatoire des } \\
\text { fonctions médico-techniques. } \\
\text { Pôle inter-établissement avec } \\
\text { délégation de gestion au niveau } \\
\text { du GHT. }\end{array}$ \\
\hline $\begin{array}{l}\text { Mode de coordination } \\
\text { des activités }\end{array}$ & $\begin{array}{l}\text { Participation aux GIE et aux GCS, } \\
\text { conventions multiples avec des } \\
\text { établissements externes à la CHT. } \\
\text { Lourdeur de la gouvernance. } \\
\text { Équipes médicales communes. } \\
\end{array}$ & $\begin{array}{l}\text { Principe hiérarchique pour fonc- } \\
\text { tion support. } \\
\text { Filières de soins et activités médi- } \\
\text { co-techniques territorialisées. } \\
\text { Équipes médicales communes. } \\
\end{array}$ \\
\hline $\begin{array}{l}\text { Degré d'intégration et } \\
\text { d'utilisation des TIC } \\
\text { et pilotage des flux }\end{array}$ & $\begin{array}{l}\text { Difficulté d'interconnexion, lo- } \\
\text { giciels différents, compétences } \\
\text { numériques variables. } \\
\text { Pilotage au cas par cas des pa- } \\
\text { tients et fuites nombreuses au } \\
\text { sein des CHT. } \\
\end{array}$ & $\begin{array}{l}\text { Convergence des systèmes d'in- } \\
\text { formation. Schéma directeur du } \\
\text { GHT. } \\
\text { Dsi et Dim de territoire communs. } \\
\text { Suivis informationnels et DMP } \\
\text { partagés. }\end{array}$ \\
\hline AUTONOMIE & $\begin{array}{c}\text { Forte mais contrariée en } \\
\text { externe }\end{array}$ & Partielle et négociée en interne \\
\hline Type d'autonomie & $\begin{array}{l}\text { Large : sur l'emploi, le dialogue } \\
\text { social, les relations patients, les } \\
\text { conventions et partenariats, les } \\
\text { projets d'investissement, les } \\
\text { activités. }\end{array}$ & $\begin{array}{l}\text { Réduite, sauf sur emplois et acti- } \\
\text { vités opérationnelles. } \\
\text { En partie préservée du fait de } \\
\text { l'absence de personnalité morale } \\
\text { du GHT. }\end{array}$ \\
\hline $\begin{array}{l}\text { Degré d'autonomie } \\
\text { dans les prises de } \\
\text { décisions }\end{array}$ & $\begin{array}{l}\text { Fort mais encadré par les acteurs } \\
\text { externes à la CHT (ARS, HAS, DGOS, } \\
\text { CRC, etc.). }\end{array}$ & $\begin{array}{l}\text { Faible sur fonctions supports, } \\
\text { partagé sur activités médicales, } \\
\text { encadré en interne par le GHT. }\end{array}$ \\
\hline
\end{tabular}




\begin{tabular}{|c|c|c|}
\hline $\begin{array}{l}\text { COMPOSANTES ET } \\
\text { INDICATEURS }\end{array}$ & $\begin{array}{c}\text { Снт-PP } \\
\text { (Observation terrain) }\end{array}$ & $\begin{array}{c}\text { GHT } \\
\text { (Analyse de la loi santé 2016) }\end{array}$ \\
\hline $\begin{array}{l}\text { Mode d'expression } \\
\text { de l'autonomie de } \\
\text { décision }\end{array}$ & $\begin{array}{l}\text { Via les assemblés de la CHT, } \\
\text { décisions souveraines de l'or- } \\
\text { donnateur de l'établissement } \\
\text { (indépendance pour les décisions } \\
\text { stratégiques de coopération hors } \\
\text { CHT). }\end{array}$ & $\begin{array}{l}\text { Via les instances communes du } \\
\text { GHT et au sein du comité stra- } \\
\text { tégique. Via le domaine de com- } \\
\text { pétences réservé des directeurs } \\
\text { d'établissement et via le pouvoir } \\
\text { conservé des CME locales. }\end{array}$ \\
\hline COHÉSION & Nécessaire et fragile & Imposée mais utile \\
\hline $\begin{array}{l}\text { Valeurs partagées et } \\
\text { degré de confiance } \\
\text { entre les partenaires }\end{array}$ & $\begin{array}{l}\text { Valeurs des personnels hospita- } \\
\text { liers (qualité des soins, service } \\
\text { public, respect des patients, al- } \\
\text { truisme). } \\
\text { Confiance indispensable pour me- } \\
\text { ner à bien des projets communs. } \\
\text { Importance du sentiment de } \\
\text { réciprocité et d'utilité de la parti- } \\
\text { cipation au réseau. }\end{array}$ & $\begin{array}{l}\text { Valeurs des personnels des éta- } \\
\text { blissements de santé. } \\
\text { Confiance utile pour le bon fonc- } \\
\text { tionnement du groupement. } \\
\text { Importance du sentiment de } \\
\text { réciprocité et d'utilité de la parti- } \\
\text { cipation au réseau. }\end{array}$ \\
\hline $\begin{array}{l}\text { Modalités de contrôle } \\
\text { et de résolution de } \\
\text { conflits }\end{array}$ & $\begin{array}{l}\text { Instance commune uniquement } \\
\text { consultative. } \\
\text { Décisions finales relevant des } \\
\text { établissements. } \\
\text { Négociation fondée sur l'adhésion } \\
\text { et le consensus. } \\
\text { Contrôle externe individualisé. }\end{array}$ & $\begin{array}{l}\text { Instance commune pouvant rece- } \\
\text { voir des délégations de pouvoir } \\
\text { des instances locales. } \\
\text { Rôle exécutif du comité straté- } \\
\text { gique et de son bureau. } \\
\text { Certification et validation } \\
\text { conjointes des documents finan- } \\
\text { ciers. } \\
\text { Contrôle externe globalisé. } \\
\end{array}$ \\
\hline $\begin{array}{l}\text { Rôles des acteurs } \\
\text { réticulaires }\end{array}$ & $\begin{array}{l}\text { Directeurs et présidents de CME, } \\
\text { élus locaux. } \\
\text { Personnalités entreprenantes. } \\
\text { Chefs de pôle des établissements. }\end{array}$ & $\begin{array}{l}\text { Directeurs et présidents de CME, } \\
\text { élus locaux, Dim de territoire, DsI } \\
\text { de GHT, chef de pôle inter-éta- } \\
\text { blissements, représentants } \\
\text { syndicaux. }\end{array}$ \\
\hline
\end{tabular}

Source : élaboration personnelle.

L'hétérogénéité est augmentée et surtout reconnue au travers du rôle particulier attribué à l'hôpital pivot. Le GHT intervient maintenant sur tous les champs du PMSI (Mco, SsR, PsY, HAD) et la présence d'un CHU lui permet en principe de couvrir l'intégralité de la gravité des soins et d'organiser en interne la graduation des prises en charge. Cette hétérogénéité issue d'une diversité supplémentaire des offreurs donne au GHT la possibilité de s'engager dans des spécialisations d'activité sur une chaîne de valeur basée sur des avantages comparatifs entre les membres. Elle reste néanmoins limitée par la nature essentiellement publique et hospitalière du regroupement ce qui ne facilite pas, même si des associations sont envisageables, la coordination autour des réseaux de soins primaires (médecine libérale et professions paramédicales), alors que ceux-ci doivent contribuer à filtrer l'accès à l'hôpital et tenir un rôle clé dans le système de santé (Pouvourville, 2016). Ce risque de « recroquevillement» du secteur public est actuellement souligné par les fédérations nationales telles que la FHF, la FHP et la CSMF (Houdart et Associés, 2016).

Les partenariats sont maintenant organisés et cartographiés afin d'être multipliés. Le GHT est une structure fédérative pour un ensemble de fonctions 
support et, en premier lieu, pour la collecte, la gestion et l'utilisation de l'information. Le directeur de l'hôpital référent devient de fait le directeur des fonctions supports déléguées. Le projet médical partagé (PMP) constitue la clé de voûte du groupement et le document stratégique autour duquel les activités devront s'organiser. La création de pôles inter-établissements est rendue nécessaire pour organiser en commun les prestations médico-techniques et elle est recommandée pour la coordination des activités cliniques. Les recrutements partagés et la mobilité intra-GHT des personnels sont encouragés par des dispositions règlementaires.

Si l'autonomie est fortement réduite sur un ensemble de compétences, elle reste importante sur un plan opérationnel pour la gestion des personnels, l'organisation des soins et les relations avec les patients. Elle disparaît de fait pour certaines fonctions supports et devient plus encadrée par le PMP et les instances communes du GHT pour les décisions stratégiques portant sur l'accroissement de capacité, les investissements importants et les conventions externes au groupement. L'examen des EPRD et des PFGP au regard de celui des autres membres introduit officiellement un encadrement collectif des décisions financières. Certes, l'autonomie des établissements hospitaliers est depuis toujours sous contrôle. Ceux-ci n'ont jamais eu ni revendiqué les capacités stratégiques des entreprises. Ce qui change avec la mise en place des GHT, c'est que cette autonomie qui était principalement encadrée par des acteurs externes (DGOS, ARS, HAS, etc.) sera maintenant en grande partie à négocier au sein du GHT et dans ses organes comme le comité stratégique, le comité des élus ou la commission médicale.

On pourrait presque dire que la cohésion n'est plus un sujet puisque l'adhésion au GHT est obligatoire et irréversible. En effet, comme le signale Brousseau (2000), le cadre institutionnel est déterminant et la confiance et les normes de comportement sont générées et maintenues par l'existence d'un cadre légal et juridique favorable et stable. Cependant, en plus du cadre juridique, c'est le partage d'un ensemble de valeurs et de normes qui favorise ces coopérations. La réciprocité, la confiance et l'identité collective sont des normes de comportement qui permettent ces coopérations (Ostrom, 1990). La loi définit un cadre potentiellement très fédératif, mais les conventions constitutives et les règlements intérieurs des instances du GHT peuvent donner naissance à des niveaux de coopération et d'intégration variables. Le rôle des acteurs réticulaires, notamment les membres du comité stratégique, les présidents de CME et les chefs de pôle seront déterminants. La confiance, le respect mutuel et le partage des mêmes objectifs de santé publique resteront des vecteurs de la cohésion d'un groupe qui devra faire des arbitrages délicats sur l'évolution de l'offre de soins des membres. Le sentiment de chacun de pouvoir profiter à son niveau de la participation au GHT conditionnera en partie la dynamique de réussite ou favorisera l'immobilisme des 135 GHT qui couvrent désormais l'intégralité du territoire national. 


\subsection{Un effet attendu sur la performance de l'hôpital public}

La volonté de rendre les acteurs publics plus efficients est une des raisons de la création des CHT puis des GHT : plus efficient pour maximiser le nombre et la qualité de la prise en charge des malades sous la contrainte d'une ressource collective commune, et plus efficient pour soutenir la concurrence avec un secteur privé engagé depuis longtemps dans une rationalisation de son offre. À ce stade et à l'aide des résultats présentés ci-dessus, nous sommes en mesure de proposer une lecture économique de l'effet potentiel des GHT sur la compétitivité des hôpitaux publics et de montrer comment ils peuvent permettre la mise en place d'une véritable stratégie de groupe pour accompagner le déplacement progressif du cadre de la concurrence.

Le GHT est une innovation institutionnelle qui vise essentiellement à diminuer à court terme la concurrence interne entre les membres du réseau pour augmenter à moyen terme la capacité concurrentielle de l'ensemble de ses membres. En tant que groupes d'offreurs, les GHT sont soumis à deux rivalités distinctes, une rivalité sur leur territoire avec les autres offreurs de soins présents et une rivalité avec les GHT implantés sur d'autres territoires. En effet, ils ne répondent que partiellement à la demande Mco ( $40 \%$ pour la CHT-PP) sur leur zone d'attractivité d'une part, et ils sont mis en concurrence implicite avec les autres GHT par la comparaison des coûts moyens de prise en charge des patients d'autre part. À l'avenir, les territoires dans lesquels les hôpitaux publics seront les mieux organisés au travers de GHT intégrés, spécialisés et coordonnés, seront aussi ceux où ils gagneront des parts de marché sur le secteur privé et obtiendront des avantages compétitifs sur les autres GHT en termes de coûts complet et de pertinence de la prise en charge des patients.

Par sa nature plus intégrative que les CHT, le GHT ouvre la possibilité de développer une véritable stratégie de groupe. Cette stratégie, qui réutilise la sémantique de l'entreprise concurrentielle (parts de marché, créneaux, maximisation du profit), est rendue possible par la vente des activités à un prix fixé (tarif des GHM) qui transforme la santé en un quasi-marché. Le système de paiement pousse alors les acteurs à réduire les coûts par patient, à augmenter les revenus générés par le patient et à augmenter le nombre de patients. Cela est réalisable par des actions portant à la fois sur des aspects liés à la maîtrise de la demande et à la maîtrise de l'offre :

- l'action sur la demande conditionne le succès dans la course inévitable aux volumes d'activité. Elle consiste, dans un domaine où la liberté de choix reste la règle, à capter des flux de patients toujours plus importants par une offre complète et diversifiée, tout en maintenant la proximité et l'attractivité de tous les membres du réseau sur le territoire. Afin de maximiser la valeur de chaque patient entrant dans le GHT, il s'agit d'accueillir, d'orienter et de conserver à l'intérieur du réseau les patients qui poursuivent ainsi des parcours de soins multi-établissements. La construction de filières de soins intégrées et la densité des liens avec les professionnels de santé extérieurs 
au GHT seront déterminantes. À cet égard, le médecin Dim de territoire devra jouer un rôle clé pour établir une cartographie et un diagnostic partagé de l'offre du groupement sur lequel sera basé le PMP, mais aussi, avec l'aide de la DsI, pour accélérer la convergence et l'interopérabilité des systèmes d'information afin de permettre une traçabilité et une continuité informationnelle ;

- l'action sur l'offre passe par la maîtrise des coûts et de la qualité. Le GHT facilitent trois types de mesures qui visent à enclencher un maximum d'économies d'échelle et d'expérience à l'intérieur du réseau et, par conséquent, lui fournir un avantage concurrentiel sur les autres GHT. Il s'agit premièrement de provoquer la baisse des coûts de prise en charge par l'amortissement des coûts fixes et la réduction des coûts variables. Un partage des équipements, des fonctions supports et de l'information sur les meilleures pratiques organisationnelles. Deuxièmement, il s'agit de piloter la chaîne de valeur multi-acteurs en imposant une spécialisation de l'activité entre les membres pour faire jouer les avantages comparatifs, éviter les redondances et optimiser l'allocation spatiale des activités. Troisièmement, afin de maintenir un maillage fin sur le territoire et une offre décentralisée qui reste attractive, le GHT pourra fournir un soutien aux éléments fragiles et vulnérables par la mise à disposition de l'ensemble du réseau des ressources rares et des compétences clés que les membres ne pourraient individuellement se procurer sur le marché.

Cette stratégie de groupe devra être adoptée et expliquée dans les différents forums et arènes du GHT en insistant sur un point qui n'est pas toujours bien perçu par les acteurs, à savoir que la performance globale du réseau dépendra du maintien de la performance de tous ses membres. Si à court terme les membres peuvent être incités à se siphonner mutuellement des parts de marché, cette concurrence de type «beggar-thy-neighbour» provoquera un affaiblissement des acteurs périphériques préjudiciable à la capacité de l'ensemble du réseau à soutenir la double rivalité, interne sur son territoire et externe avec les autres territoires.

\section{Conclusion}

Les quatre dimensions retenues pour l'analyse des réseaux publics d'offre de soins nous permettent de décrire l'évolution récente du cadre institutionnel imposé aux hôpitaux publics, notamment la transformation des CHT en GHT. Par rapport à la $\mathrm{CHT}$, le GHT voit son hétérogénéité augmentée même si celle-ci reste insuffisante et ne concerne que les établissements publics. Les partenariats sont imposés et organisés avec la volonté de privilégier les projets internes au groupement. L'autonomie des membres est fortement réduite et devra être négociée dans les différents organes du groupement par l'intermédiaire des acteurs réticulaires. Enfin, la cohésion qui est renforcée par le législateur n'est plus une condition existentielle, mais déterminera en partie l'efficience et l'efficacité à venir du groupement. Le GHT reste un compromis institutionnel fragile 
entre la fusion-absorption non souhaitée et l'éparpillement des souverainetés non tenables. Le cadre juridique est assez souple pour donner naissance à des niveaux d'intégration variables, selon les contingences locales, pour toujours rechercher une meilleure réponse aux besoins de santé. Dans ce contexte, la validité interne de notre recherche est, comme souligné, principalement favorisée par la triangulation des données, la saturation empirique du terrain, mais aussi par la restitution pour validation des résultats, post-analyse, aux acteurs clés de la CHT-PP. Quant à la validité externe, elle repose sur une constance des contextes car les CHT comme les GHT ont les mêmes règles juridiques, les mêmes statuts de personnel, les mêmes tutelles et les mêmes principes d'organisation. Ainsi, les résultats de l'étude de la Chт-Pि peuvent, avec quelques précautions en termes de poids des établissements et de nature du réseau (présence d'un établissement central, GHT bilatéral, poids et nombre des hôpitaux périphériques, proximité géographique des établissements, etc.), être généralisables et transférables à d’autres GHT.

Le GHT était en gestation dans les grands rapports, tels que la stratégie nationale de santé de 2013 ou le Rapport Devictor de 2014 visant à « développer l'approche territoriale et populationnelle de l'offre en santé». C'est par les acteurs publics, sur lesquels l'État a directement la main, que le gouvernement a imposé des regroupements territoriaux pour inciter à la coopération autour de la prise en charge des patients. Paradoxalement, le renforcement des GHT ne risquet-il pas de maintenir les défauts d'un système fracturé dans lequel l'ensemble des intervenants sur un même territoire restent cloisonnés et répondent à des logiques financières et de fonctionnement différents ? La médecine de ville, les établissements privés et les acteurs médico-sociaux peuvent développer des partenariats avec les GHT, mais n'en sont pas membres à part entière. C'est d'un rapprochement entre les GHT et les Commissions Professionnelles Territoriales de Santé (CPTS) qu'un véritable réseau complet d'acteurs territoriaux pourrait naître, un modèle proche des «health systems» américains, permettant une contractualisation avec les ARs pour une prise en charge quasi-intégrale des territoires (Pouvourville, 2016). Les GHT et les CPTS seront amenés à se rapprocher pour avancer sur la mise en place encore inachevée d'un Service Public Territorial de Santé, et cela d'autant plus que c'est au niveau des soins de premier recours, où l'hôpital public n'est pas dominant, que la coordination des acteurs est le plus nécessaire. Pour ce faire, d'autres réformes règlementaires sont certainement indispensables, notamment sur les modes de financement des structures et des cycles de soins, mais aussi sur le statut des personnes pour faciliter et organiser les mobilités de l'ensemble des professionnels de santé.

\section{Bibliographie}

AMAT-ROZE J.-M. (2011), « La territorialisation de la santé : quand le territoire fait débat», Revue Hérodote - Éd. La Découverte, Vol. 4, No. 143, p. 13-32. 
ANAP (2011), Guide méthodologique des coopérations territoriales, Ministère du Travail, de l'Emploi et de la Santé, République Française, Paris. Vol. 2, Janvier [www.anap.fr].

ASSENS C. (2013), Le management des réseaux, De Boeck, Bruxelles.

AUBERT B., PATRY M., RIVARD S. (1999), «L'organisation virtuelle», in : Poitevin M. (dir.), Impartition : fondements et analyses, PUL, Québec, p. 243-263.

BALY O., CAZIN L., DESPATIN J., KLETZ F., PERIAC E. (dir.) (2016), Management hospitalier et territoires : les nouveaux défis, Presses des Mines, Paris.

BIRONNEAU L., VIVIANI J.-L. (dir.) (2016), Collaborations et réseaux Approches transversales en management, Presses Universitaires de Rennes (PUR), Rennes.

BROUSSEAU É. (2000), «La gouvernance des processus de coopération », in : Voisin C., Plunket A., Bellon B. (dir.), La coopération industrielle, Économica, Paris, p. 29-43.

BRUYÈRE C., VERLAQUE A. (2009), «Un élargissement du concept de réseau clignotant : proposition de lecture de deux formes de réseaux territorialisés », Management \& Avenir, No. 24, p. 178-195.

CARLES J. (2006), «Réseau, stratégie et gouvernance», L'Expansion Management Review, Vol. 4, No. 123, p. 85-95.

CAZIN L. (2016), «Coopérations territoriales : un nouvel outil stratégique au service de l'hôpital-entreprises ? », Chapitre 7 in 0 . Baly et al. (dir.), Management hospitalier et territoires : les nouveaux défis, Presses des Mines, Paris, p. 175-194.

CLÉMENT C. (2012), La Communauté hospitalière de territoire (Cht), mode d'emploi, Éd. Les Études Hospitalières (Pratiques professionnelles), Bordeaux.

COLDEFY M., LUCAS-GABRIELLI V. (2010), «Les territoires de santé : des approches régionales variées de ce nouvel espace de planification », Pratiques et Organisation des Soins, Vol. 41, No. 1, p. 73-80.

COLDEFY M., LUCAS-GABRIELLI V. (2012), « Le territoire, un outil d'organisation des soins et des politiques de santé? », Questions d'économie de la santé, Irdes, Avril, No. 175, p. 1-8.

DUMEZ H. (2013), Méthodologie de la recherche qualitative, Vuibert, Paris.

DUSSUC B., Geindre S. (2015), «Intermédiation et clusterisation », Chapitre 7 in C. Pardo et G. Paché (dir.), Commerce de gros et commerce inter-entreprises, Éd. Ems, Caen, p. 145-156.

EHLINGER S., PERRET V., CHABAUD D. (2007), « Quelle gouvernance pour les réseaux territorialisés d'organisations? », Revue Française de Gestion, Vol. 33, No. 170, p. 155-171. 
FERMON B., GRANDJEAN P. (2015), Performance et innovation dans les établissements de santé, Dunod, Paris.

FRÉRY F. (1998), « Les réseaux d'entreprises : une approche transactionnelle », in : Laroche H., Nioche J.-P. (dir.), Repenser la stratégie - Fondements et perspectives, Vuibert, Paris, p. 61-84.

FULCONIS F. (2004), « Le réseau, objet de recherche en gestion. La pluralité des cadres d'analyse ", Chapitre 3 in C. Voisin et al. (dir.), Les réseaux : dimensions stratégiques et organisationnelles, Collection « Recherche en Gestion », Économica, Paris, p. 59-75.

GAVARD-PERRET M.-L., GOTTELAND D., HAON C., JOLIBERT A. (2012), Méthodologie de la recherche. Réussir son mémoire ou sa thèse en sciences de gestion, 2e éd., Pearson France, Montreuil.

GIORDANO Y. (dir.) (2003), Conduire un projet de recherche - Une perspective qualitative, Éd. Ems, Caen.

GOMBAULT A. (2005), «La méthode des cas », Chapitre 2 in P. Roussel et F. Wacheux (dir.), Management des ressources humaines - Méthodes de recherche en sciences humaines et sociales, De Boeck, Bruxelles, p. 31-64.

GRAWITZ M. (2000), Méthodes des sciences sociales, 11e éd., Dalloz, Paris.

GRENIER C. (2014), «Proposition d'un modèle d'espaces favorables aux habiletés stratégiques. Introduction au numéro thématique : 'Les organisations de santé et leurs environnements institutionnels : réceptacles d'injonctions ou acteurs stratégiques ?' », Journal de Gestion et d'Économie Médicales, Vol. 32, No. 1, p. 3-10.

GRENIER C., GUITTON-PHILIPPE S. (2011), « La question des regroupements / mutualisations dans le champ sanitaire et social : l'institutionnalisation d'un mouvement stratégique ?», Management \& Avenir, No. 47, p. 98-113.

HLADY-RISPAL M. (2015), «Une stratégie de recherche en gestion - L'étude de cas », Revue Française de Gestion, Vol. 41, No. 253, p. 251-266.

HOUDART ET ASSOCIÉS (2016), Les GHT sonnent-ils le glas d'une politique territoriale associant l'ensemble des acteurs? Synthèse des débats du $1^{\text {er }}$ décembre, Université Paris Descartes, Paris.

HUSSER J. (2005), « Contextualisme et recueil de données », Chapitre 3 in P. Roussel et F. Wacheux (dir.), Management des ressources humaines - Méthodes de recherche en sciences humaines et sociales, De Boeck, Bruxelles, p. 65-100.

KINDLEBERGER C. (1986), "International public goods without international government”, American Economic Review, Vol. 76, No. 1, p. 1-13.

LE MENN J., MILON A. (2012), Rapport d'information sur le financement des établissements de santé, Sénat, No. 703] [en ligne : http ://www.senat.fr/ rap/r11-703/r11-7031.pdf]. 
LOUAZEL M., KELLER C. (2016), « Entre concurrence et coopération : un 'effet bloc' des incitations réglementaires sur les relations entre établissements de santé ?», Chapitre 8 in 0. Baly et al. (dir.), Management hospitalier et territoires : les nouveaux défis, Presses des Mines, Paris, p. 195-218.

MILES M., HUBERMAN A. (2003), Analyse des données qualitatives, 2e éd., De Boeck, Bruxelles.

MILES R.-E., SNOW C.-C. (1986), "Network organizations : new concepts for new forms", The McKinsey Quarterly, Autumn, p. 53-66.

NORTH D. (1990), Institutions, Institutional Change and Economic Performance, Cambridge University Press.

OSTROM E. (1990), Governing the commons. The evolution of institutions for collective action, Cambridge University Press, Cambridge.

PACHÉ G., PARAPONARIS C. (2006), L'entreprise en réseau : approches inter et intra-organisationnelles, Éd. de l'Adreg [en ligne : http ://thierry-verstraete. com/editions-de-ladreg].

PIORE M., SABEL C. (1984), The second industrial divide: possibilities for prosperity, Basic Books, New York.

PORTER M. (2000), “Location, competition and economic development: local clusters in a global economy", Economic Development Quarterly, Vol. 14, No. 1, p. 15-34.

POUVOURVILLE (de) G. (2016), «Le territoire dans la régulation du système de santé », Préambule in 0 . Baly et al. (dir.), Management hospitalier et territoires : les nouveaux défis, Presses des Mines, Paris, p. 13-25.

ROYER I., ZARLOWSKI P. (2014), «Échantillon(s)», Chapitre 8 in R.A. Thiétart et al., Méthodes de recherche en management, 4 e éd., Dunod, Paris, p. 188-223.

RUILLER C., BURELLIER F. (2016), «Un médecin, un soignant et un gestionnaire sont dans un bateau... la compétence collective au sein des pôles hospitaliers », in L. Bironneau et J.-L. Viviani (Dir.), Collaborations et réseaux, Presses Universitaires de Rennes, Rennes, p. 47-63.

SALAUN V. (2014), «Organisation Virtuelle Éphémère, quelle place pour le management logistique? Le cas des festivals musicaux », Logistique \& Management, Vol. 22, No. 2, p. 61-69.

SAMPIERI-TEISSIER N., ROLLIN F. (2015), «Pour une meilleure intégration des services logistiques - Limites et avenues des possibles », Gestions Hospitalières, No. 542, p. 16-21.

SNOW C.-C., MILES R.-E., COLEMAN H.-J. (1992), "Managing 21st century network organizations”, Organizational Dynamics, Vol. 20, No. 3, p. 5-20. 
SUIRE R., VICENTE J. (2008), « Théorie économique des clusters et management des réseaux d'entreprises innovantes », Revue Française de Gestion, Vol. 34, No. 184, p. 119-136.

TABUTEAU D. (2010), « Loi 'Hôpital, patients, santé et territoires' (HPST) : des interrogations pour demain! », Santé Publique, Vol. 22, No. 1, p. 78-90.

THIÉTART R.-A. et al. (2014), Méthodes de recherche en management, 4e éd., Dunod, Paris.

THORELLI H.-B. (1986), "Networks : between markets and hierarchies", Strategic Management Journal, Vol. 7, No. 1, p. 37-51.

TURNER J.-R., MÜLLER R. (2003), “On the nature of the project as a temporary organization", International Journal of Project Management, Vol. 21, No. 1, p. 1-8.

VAN CAMPENHOUDT L., QUIVY R. (2011), Manuel de recherche en sciences sociales, 4e éd., Dunod, Paris.

VOISIN C., BEN MAHMOUD-JOUINI S., ÉDOUARD S. (dir.) (2004), Les réseaux: dimensions stratégiques et organisationnelles, Économica, Paris.

WACHEUX F. (1996), Méthodes qualitatives et recherche en Gestion, Économica, Paris.

WARNOTTE G., ROUSSEAU A. (1993), « La responsabilisation, un construit social. Transformation du rôle du management dans l'implantation d'un projet qualité totale : contribution d'une lecture contextuelle », Actes du 4e congrès de l'AGRH, Jouy-en-Josas, p. 430-437.

WILLIAMSON O. (1981), "The modern corporation : origins, evolution, attributes", Journal of Economic Literature, December, No. 29, p. 1537-1568.

YIN R. (2009), Case study research: design and methods, 4th ed., Sage Publications, London. 